\title{
A NOTE ON MEDIAL DIVISION GROUPOIDS
}

\author{
J. JEŽEK AND T. KEPKA
}

(Communicated by Maurice Auslander)

\begin{abstract}
In 1949 Sholander showed that every medial cancellation groupoid can be embedded into a medial quasigroup. In this note we prove the dual assertion that every medial division groupoid is a homomorphic image of a medial quasigroup.
\end{abstract}

\section{INTRODUCTION}

By a groupoid we mean a nonempty set with one binary operation, for which we use the multiplicative notation as a default. A groupoid is called medial (in some papers entropic, in [4] alternation) if it satisfies the identity

$$
(x y)(u v)=(x u)(y v) .
$$

While [2] can serve as a reference on the theory of medial groupoids, the book [3] gives numerous examples and connections with other parts of mathematics. Given a groupoid $G$ and an element $a \in G$, the left translation $L_{a}$ of $G$ is the mapping of $G$ into itself defined by $L_{a}(x)=a x$ for any $x \in G$. Similarly, the right translation $R_{a}$ is defined by $R_{a}(x)=x a$. We say that $G$ is a cancellation groupoid if all its translations are injective mappings. If all the translations are surjective, $G$ is a division groupoid. A quasigroup is a cancellation and division groupoid.

As it is easy to see, a homomorphic image of a division groupoid is a division groupoid. In particular, a homomorphic image of a medial quasigroup is a medial division groupoid. The aim of this paper is to prove that each medial division groupoid can be obtained as a homomorphic image of a medial quasigroup.

Our proof will be based on an auxiliary construction given in $\S 2$ which is, in fact, a two-dimensional version of the ergodic-theoretic construction of an automorphism on a measure space naturally extending an endomorphism (see $[1$, Chapter $10, \S 4]$ for the entropic theory of dynamical systems).

Let us remark that, according to [2, Proposition 6.4.1], finitely generated medial division groupoids are already quasigroups.

For a groupoid $G$ we define a binary relation $t_{G}$ on $G$ by $(a, b) \in t_{G}$ iff $L_{a}=L_{b}$ and $R_{a}=R_{b}$. Clearly, $t_{G}$ is a congruence of $G$.

Received by the editors July 17, 1991 and, in revised form, March 5, 1992.

1991 Mathematics Subject Classification. Primary 20N05, 08A05.

Key words and phrases. Medial groupoid, quasigroup, division. 
A groupoid $G$ is said to be regular if for any $a, b, c \in G, a c=b c$ implies $L_{a}=L_{b}$ and $c a=c b$ implies $R_{a}=R_{b}$. Clearly, every cancellation groupoid is regular. Both the class of cancellation groupoids and the class of regular groupoids are quasivarieties.

From [2] we shall need the following two results.

Lemma 1. Let $G$ be a medial division groupoid. Then the factor $G / t_{G}$ is regular. Proof. See [2, Lemma 6.2.3].

Lemma 2. Let $G$ be a regular medial division groupoid. Then there exist an abelian group $G(+)$, two commuting surjective endomorphisms $f, g$ of $G(+)$, and an element $q \in G$ such that

$$
x y=f(x)+g(y)+q
$$

for all $x, y \in G$.

Proof. See [2, Corollary 6.1.2].

\section{BI-UNARY ALGEBRAS: AN AUXILIARY CONSTRUCTION}

Lemma 3. Let $S$ be a nonempty set and $f, g$ be two commuting surjective transformations of $S$. Then there are a set $A$, two commuting permutations $F, G$ of $A$, and a mapping $\varphi$ of $A$ onto $S$ such that $\varphi F=f \varphi$ and $\varphi G=g \varphi$. Proof. Let $N$ denote the set of positive integers. Denote by $A$ the set of the mappings $a: N \times N \rightarrow S$ such that

$$
f(a(i+1, j))=g(a(i, j+1))=a(i, j)
$$

for all $i, j \in N$. (It is possible to imagine the elements of $A$ as being infinite matrices over the set $S$.) For $a \in A$ define elements $F(a)$ and $G(a)$ of $A$ by

$$
F(a)(i, j)=f(a(i, j)), \quad G(a)(i, j)=g(a(i, j)) .
$$

With respect to $f g=g f$, it is easy to check that both $F(a)$ and $G(a)$ belong to $A$ for any $a \in A$. The mappings $F, G$ commute, as

$$
F G(a)(i, j)=f g(a(i, j))=g f(a(i, j))=G F(a)(i, j) .
$$

We are going to show that $F$ is a permutation of $A$. If $a, b \in A$ are elements such that $F(a)=F(b)$, then for all $i, j \in N$ we have

$$
\begin{aligned}
a(i, j) & =f(a(i+1, j))=F(a)(i+1, j) \\
& =F(b)(i+1, j)=f(b(i+1, j))=b(i, j)
\end{aligned}
$$

and consequently $a=b$. Given an element $c \in A$, we can define $d$ by $d(i, j)=c(i+1, j)$ for all $i, j$ and check that $d \in A$ and $F(d)=c$.

In the same way one can prove that also $G$ is a permutation of $A$. Define a mapping $\varphi: A \rightarrow S$ by $\varphi(a)=a(1,1)$. For all $a \in A$ we have

$$
\varphi F(a)=F(a)(1,1)=f(a(1,1))=f \varphi(a)
$$

and thus $\varphi F=f \varphi$. Similarly, $\varphi G=g \varphi$. It remains to show that $\varphi$ is a mapping onto $S$. 
Let $s$ be an arbitrary element of $S$. Put $a_{1,1}=s$ and for any $i \geq 2$ choose an element $a_{i, i} \in S$ such that $f g\left(a_{i, i}\right)=a_{i-1, i-1}$; this is possible, as $f g$ is surjective. Setting

$$
a(i, j)= \begin{cases}g^{i-j}\left(a_{i, i}\right) & \text { for } i \geq j, \\ f^{j-i}\left(a_{j, j}\right) & \text { for } i<j,\end{cases}
$$

we obtain a mapping $a$ of $N \times N$ into $S$. We only need to prove that $a \in A$, since $\varphi(a)=s$ will then follow from our choice $a_{1,1}=s$. If $i \geq j$, then

$$
f(a(i+1, j))=f g^{i+1-j}\left(a_{i+1, i+1}\right)=g^{i-j}\left(a_{i, i}\right)=a(i, j) .
$$

If $j=i+1$, then

$$
f(a(i+1, j))=f\left(a_{i+1, i+1}\right)=f\left(a_{j, j}\right)=a(j-1, j)=a(i, j) .
$$

If $j>i+1$, then

$$
f(a(i+1, j))=f^{j-i}\left(a_{j, j}\right)=a(i, j) .
$$

We have proved $f(a(i+1, j))=a(i, j)$ in all cases, and $g(a(i, j+1))=a(i, j)$ can be checked similarly.

Remark. Although we shall not use the fact in the following, let us remark that the construction of $A, F, G, \varphi$ given in the proof of Lemma 3 is universal in the sense that if $A_{1}, F_{1}, G_{1}, \varphi_{1}$ is any other quadruple with the same properties, then there exists a uniquely determined mapping $\psi: A_{1} \rightarrow A$ such that $\psi F_{1}=F \psi$ and $\psi G_{1}=G \psi$.

\section{MEDIAL DIVISION GROUPOIDS: THE MAIN RESULT}

Lemma 4. Let $G$ be a medial division groupoid. Then $G$ is a homomorphic image of the regular medial division groupoid $G / t_{G} \times G / t_{G}$.

Proof. Let $\varphi: G \rightarrow G / t_{G}$ be the canonical projection. It follows from the definition of $t_{G}$ that $\psi: G / t_{G} \times G / t_{G} \rightarrow G$ is a correctly defined mapping if we put $\psi(\varphi(x), \varphi(y))=x y$ for all $x, y \in G$. By the medial law,

$$
\begin{aligned}
\psi((\varphi(x), \varphi(y)) \cdot(\varphi(u), \varphi(v))) & =(x u)(y v)=(x y)(u v) \\
& =\psi(\varphi(x), \varphi(y)) \cdot \psi(\varphi(u), \varphi(v))
\end{aligned}
$$

for any $x, y, u, v \in G$ and we see that $\psi$ is a homomorphism. Since $G$ is a division groupoid, $\psi$ is surjective. The factor $G / t_{G}$ is a regular medial division groupoid by Lemma 1 , and it is clear that the product of regular medial division groupoids is a regular medial division groupoid.

Theorem 5. Every medial division groupoid is a homomorphic image of a medial quasigroup.

Proof. With respect to Lemma 4, it is sufficient to prove that any regular medial division groupoid $G$ is a homomorphic image of a medial quasigroup. By Lemma 2 there are an abelian group $G(+)$, two commuting surjective endomorphisms $f, g$ of $G(+)$, and an element $q \in G$ such that $x y=f(x)+g(y)+q$ for all $x, y \in G$. By Lemma 3 there exist a set $A$, two commuting permutations $F, G$ of $A$, and a mapping $\varphi$ of $A$ onto $G$ such that $\varphi F=f \varphi$ and 
$\varphi G=g \varphi$. Denote by $H(+)$ the free abelian group over the set $A$. The permutations $F, G$ can be uniquely extended to automorphisms $\alpha, \beta$ of $H(+)$, and we have $\alpha \beta=\beta \alpha$. Moreover, the mapping $\varphi$ can be extended to a homomorphism $h$ of $H(+)$ onto $G(+)$. Since the homomorphisms $h \alpha$ and $f h$ of $H(+)$ into $G(+)$ coincide on the set of generators $A$, they coincide everywhere and we have $h \alpha=f h$. Similarly, $h \beta=g h$. Take an element $e \in H$ such that $h(e)=q$ and define a multiplication on $H$ by $x y=\alpha(x)+\beta(y)+e$. Then $H$ becomes a medial quasigroup, and one can easily verify that $h$ is a homomorphism of the quasigroup $H$ onto the groupoid $G$.

Remark. For a given medial division groupoid $G$ let $Q$ be a medial quasigroup and $r$ be a congruence of $Q$ such that $G \simeq Q / r$. Among the congruences $s$ of $Q$ such that $s \subseteq r$ and $Q / s$ is a quasigroup, there is a unique largest one; denote it by $s_{0}$. Then $G$ is a homomorphic image of the medial quasigroup $Q_{0}=Q / s_{0}$ with the property that no nontrivial congruence of $Q_{0}$ contained in the kernel of the homomorphism factors $Q_{0}$ to a quasigroup. In this sense, every medial division groupoid has a "quasigroup cover". We do not know, however, if this medial quasigroup cover is unique.

\section{REFERENCES}

1. I. P. Cornfeld, S. V. Fomin, and Ya. G. Sinai, Ergodic theory, Grundlehren Math. Wiss., vol. 245, Springer-Verlag, New York, 1982.

2. J. Ježek and T. Kepka, Medial groupoids, Rozpravy ČSAV, Řada Mat. a Přír. Věd 93/2, Academia, Praha, 1983.

3. A. Romanowska and J. D. H. Smith, Modal theory-an algebraic approach to order, geometry and convexity, Helderman Verlag, Berlin, 1985.

4. M. Sholander, On the existence of the inverse operation in alternation groupoids, Bull. Amer. Math. Soc. 55 (1949), 746-757.

Department of Mathematics, University of Hawail, Honolulu, Hawail 96822

E-mail address: jarda@kahuna.math.hawaii.edu

Department of Mathematics, Charles University, Sokolovská 83, Praha 8, 18600 Czech Republic 